\title{
Perceived depth vs. structural relevance in the object-superiority effect
}

\author{
MARK LANZE and NAOMI WEISSTEIN \\ State University of New York, Buffalo, New York \\ and \\ JUDITH RICH HARRIS \\ New Providence, New Jersey
}

\begin{abstract}
When subjects must identify a barely visible line in a briefly flashed display, their accuracy depends on the configuration of the context in which the target line appears. Weisstein and Harris (1974) found that accuracy is highest when the target is part of a pattern that resembles a unified, three-dimensional object, and lowest in a flat-looking pattern composed of disconnected lines; they labeled this phenomenon the object-superiority effect. In the three experiments reported here, identification accuracy was found to correlate highly and significantly $(r=$ .78) with the judged depth of the patterns. Judged structural relevance of the target line to the pattern (McClelland \& Miller, 1979) was uncorrelated with accuracy $(r=-.28)$. Even when the target line appeared as an isolated fragment within the context pattern, a pattern perceived as three-dimensional yielded higher identification accuracy than one perceived as flat.
\end{abstract}

In 1974, Weisstein and Harris reported a phenomenon that they labeled the object-superiority effect. In their experiment, subjects judged which of four possible target lines was present in briefly flashed displays. When the target line was embedded in a context of other lines, identification accuracy was found to depend on the configuration of the context: it was highest when the target was part of a pattern that resembled a unified, three-dimensional object; it was lowest in a flat-looking pattern composed of disconnected lines. These context patterns were the same for all four target lines, so the differences in accuracy could not be attributed to cues provided by the contexts. Thus, these findings imply that the perception of a line in a pattern is facilitated when the pattern is object-like.

Of course, identification of a barely visible line in a briefly flashed display does not depend solely on the configuration of its context-a number of other factors may influence accuracy. For example, Earhard (1980) and Earhard and Armitage (1980) have shown that the precise location of the fixation point, relative

This research was supported in part by National Eye Institute Grants EY03047 and EY03432 and by DARPA Contract MDA 903-81-C-0320 to the second author. We thank Mary C. Williams for her suggestions and assistance, and referee Ira Bernstein for his astute and valuable comments. Thanks also go to Robert Dunford and to Francine Frome for their help with data analysis. Finally, we would like to thank William Maguire and Victor Klymenko for their helpful comments throughout the production of this paper. Address correspondence to: Naomi Weisstein, Department of Psychology, SUNY at Buffalo, 4230 Ridge Lea Road, Buffalo, New York 14226. to the lines and spaces of the display, has a noticeable effect on accuracy. In their experiments accuracy declined when the fixation point was moved from an empty space to a line or an area of converging lines. Another factor is the location of the target line relative to other lines in the display (what A. Williams \& Weisstein, 1977, have called the "local environment" of the target line). A target that forms an outside boundary of a pattern is identified more accurately than one that is surrounded by other lines (McClelland \& Miller, 1979; Wandmacher, Note 1).

A fourth determinant of identification accuracy is the total number and length of lines in the display. In general, accuracy declines as total line length goes up (McClelland \& Miller, 1979, Experiment 1; Weisstein \& Harris, 1974). This is not surprising, since increasing the number and length of nontarget lines decreases the signal-to-noise ratio. What is surprising is that the effect of context configuration may be strong enough to override the effect of the number of lines: in some experiments, a line embedded in a favorable context is identified even more accurately than a line alone (A. Williams \& Weisstein, 1978). This finding has been called the object-line effect. Whether this so-called "enhancement" effect will occur in any given experimental situation is difficult to predict. Since the negative effect of the extra lines in the display is pitted against any positive effect of context, the outcome is uncertain and easily influenced by other variables.

In contrast, the object-superiority effect is a robust and reliable phenomenon, one that has been replicated many times in a number of different labora- 
tories (see Weisstein \& Harris, 1980, for a summary). When total line length and the local environment of the target line and the fixation point are held constant, there is no question that certain types of contexts produce greater accuracy than other types of contexts. However, the favorable patterns differ from the unfavorable ones in a number of ways, and it is not clear which of the attributes of the favorable patterns is responsible for the effect. Two qualities of such patterns have previously been considered: (1) their connectedness, or how unified they appear; and (2) the amount of depth or three-dimensionality viewers perceive in them. Evidence suggests that both of these qualities play some role in object-superiority effects (Genter \& Weisstein, 1980; Weisstein \& Maguire, 1978; Weisstein, M. C. Williams, \& A. Williams, 1979; Ozog, Note 2); however, perceived depth appears to be more important than perceived connectedness (Weisstein, M. C. Williams, \& Harris, in press). Patterns that are rated high in apparent depth generally produce greater accuracy in the line identification task than patterns perceived as flat.

Recently, McClelland and Miller (1979) have argued that the crucial factor in object superiority is not the perceived depth of the pattern, but rather the "structural relevance" of the target line to the pattern as a whole. In other words, they believe that what matters is how important the target line is to the overall structure of the pattern.

McClelland and Miller reported two experiments in support of this hypothesis. In one (their Experiment 2), they found that a pattern judged to have a structurally relevant target line yielded higher accuracies than one with an irrelevant target line. However, as these experimenters noted, the pattern with the irrelevant target was also rated less threedimensional in appearance. Since the two factors covaried, either factor (or both) could have been responsible for the accuracy difference.

In their third experiment, McClelland and Miller introduced a new figure, the "hurdle," and showed that a relevant target line in the hurdle pattern was identified more accurately than an irrelevant line in the Weisstein and Harris (1974) "overlapping squares" figure. But this experiment is also inconclusive, since perceived depth and structural relevance were again confounded. Although McClelland and Miller did not obtain depth and structural relevance ratings for the hurdle figure, our informal observations suggest that viewers see it as more threedimensional than the overlapping squares pattern. Moreover, total line length was less for the hurdle pattern than for the overlapping squares, and the target was an outside line in the hurdle and an inside line in the overlapping squares.

Another study that attempted to assess the relative importance of perceived depth and perceived structural relevance of the target was reported by Weisstein et al. (in press). By using patterns that were approximately equal in total line length and in the local environment of the target line, they controlled for the effects of these extraneous factors. Unfortunately, in their experiments, too, judged depth and judged structural relevance tended to vary together. In fact, ratings of depth and of structural relevance were highly and significantly correlated, and Weisstein et al. ended by questioning whether it is possible to vary these two attributes independently.

The present experiments are an attempt to do just that. We have endeavored to construct sets of patterns in which apparent depth and apparent structural relevance of the target line are uncorrelated. Another requirement was that these patterns had to meet the two important criteria of equal total line length and approximately equal local environment of the target line. The fixation point was always located in an empty space, rather than on a line.

In these experiments, the subjects' task was to judge which of two diagonal target lines was present in a brief display. The lines were presented either alone or as part of a pattern; the lines-alone condition serves as a standard condition so that variations in accuracy can be compared across experiments. The present concern is not with the accuracy differences from the lines-alone condition, but with accuracy differences from one pattern to another. We are asking: Are these variations in accuracy related primarily to the structural relevance of the target line or are objectlike qualities-specifically, apparent depth-more important?

Ratings of apparent depth and apparent structural relevance (SR) of the target line were obtained for each pattern; a separate group of subjects made these ratings. Although using one group of subjects for the identification task and a second group for the rating task somewhat complicates statistical treatment of the data, it is a necessary precaution. Otherwise, performance on the identification task might influence a subject's ratings or vice versa.

\section{EXPERIMENT 1}

The three context patterns used in this experiment are shown in the first two columns of Figure 1. Pattern $A$ is similar to one of the overlapping squares figures of Weisstein and Harris (1974); the difference is that the target line is now an isolated segment, disconnected from the rest of the pattern.

\section{Method}

Ten subjects, naive as to the purpose of the experiment, rated the six patterns shown in Figure 1 for perceived depth and for perceived SR (structural relevance) of the target lines. Four different subjects performed the line identification task, judging which of the two possible target lines (row D in Figure 1) appeared in each briefly presented display. Some of these subjects had previously served in similar experiments; however, all but one of them were 
1 2

3
Depth
Rating

a
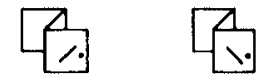

b
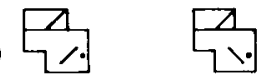

c 「少!

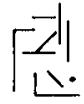

2.3

4.0

2.6

$\begin{array}{cc}\text { Structural } & \text { Accuracy } \\ \text { Relevance } & \text { (Percent } \\ \text { Rating } & \text { Correct) }\end{array}$

2.2

67

49

d ノ.
$-$

Figure 1. The patterns used in Experiment 1 are shown in columns 1 and 2, rows $A, B$, and C. Row D shows the target lines alone; the subjects' task was to judge which of these two lines was present in each brief display. Columns 3 and 4 give the mean ratings, for each context pattern, of depth and of structural relevance of the target line; these are averaged across subjects and across the two target line positions. Column 5 gives mean accuracy on the line identification task, averaged over subjects and target positions.

unaware of the purpose of this experiment. All 14 observers had normal or corrected-to-normal vision.

The rating task. The six patterns (with target lines) shown in Figure 1 were drawn in black on a single sheet of white typing paper and arranged in random order. They varied in size from 1.5 to $1.6 \mathrm{~cm}$ in width and from 1.5 to $2.2 \mathrm{~cm}$ in height. Written instructions accompanied these stimuli, directing the subjects to rate the patterns for perceived three-dimensionality and for the perceived SR of their target lines; the order of these two tasks was determined randomly.

The patterns were rated against a standard figure that was designed to be particularly high in both perceived depth and perceived SR of its component lines. The standard figure used for both kinds of ratings was a drawing of a pyramid: four sides (each equilateral triangles) on a square base. Only the front side and the right side of this figure were visible; the right side was shaded and drawn in perspective. A fixation point was located in the center of the figure.

For the depth ratings, subjects were instructed to give a pattern a rating of 10 if it appeared as three-dimensional as the standard. Lower ratings were to be used for figures that appeared less threedimensional, down to 1 for a pattern that appeared perfectly flat. The subjects were asked to gaze at the fixation points while making their judgments.

For the SR ratings, the line forming the left side of the front face of the pyramid was used as the standard. The subjects were told that this line represents a SR rating of 10 , indicating that it is highly relevant to the figure- "it is not incidental, and the figure would look very different without that line." If the target line in a pattern appeared as structurally relevant as the standard line, it was to receive a rating of 10 ; otherwise, lower ratings should be used, down to 1 for a line that was completely irrelevant to the figure.

The line identification task. The stimuli shown in Figure 1 were presented as bright lines on a dark CRT screen, for a display duration of $20 \mathrm{msec}$. The patterns were the same size as those used in

the rating task; in visual angle, they subtended from .86 to $.92 \mathrm{deg}$ horizontally and from .86 to 1.25 deg vertically. The diagonal target lines were each $.58 \mathrm{~cm}$ in length (.32 deg of visual angle). To facilitate fixation, the fixation point was slightly brighter than the target and context lines and was present continuously. No pre- or postmasks were used.

Subjects were instructed to gaze at the fixation point before and during display presentation. A chinrest maintained viewing distance at $1 \mathrm{~m}$. Subjects initiated each display presentation by pressing a foot switch. They were instructed to judge which of the two target lines was present in the display and to indicate their judgment by depressing key 2 or 3 of a Decwriter typewriter. Feedback was provided by an auditory tone. A PDP-11 computer controlled stimulus presentation and recorded responses.

The task was carried out in a darkened room. Subjects darkadapted for $10 \mathrm{~min}$ and were then given $15 \mathrm{~min}$ of practice. During the practice period, display illumination was adjusted to produce an overall accuracy level of approximately $70 \%$ to $75 \%$ correct. The luminance levels for the different subjects ranged from 3.0 to $3.5 \mathrm{~cd} / \mathrm{m}^{2}$.

Each subject completed a total of 192 experimental trials -48 for each context pattern and 48 for lines alone-in random order. The two target lines appeared equally often. Rest periods were given after every 64 trials.

\section{Results}

Ratings. Mean depth and SR ratings (averaged over the 10 subjects and the two target positions) are shown in columns 3 and 4 of Figure 1. Single-factor (repeated-measures) analyses of variance (ANOVAs) were performed on these ratings. A significant main effect of pattern was found for the depth ratings $[F(2,18)=35.6, p<.001] ;$ Newman-Keuls tests showed that each of the three patterns differed significantly from the other two in rated depth. The main effect for the SR ratings was not significant $[F(2,18)$ $=1.1]$.

Two-factor analyses of covariance ${ }^{1}$ showed that when the large differences in rated depth were statistically adjusted out, a small but significant effect of pattern on SR ratings was found $[F(5,47)=2.8$, $p<.05]$. This effect went in the direction opposite to the depth ratings. Of course, when rated SR was statistically controlled, the sizable effect of pattern on rated depth remained significant $[F(5,47)=31.7$, $\mathrm{p}<.001]$.

Accuracy. Mean accuracy scores for the three context patterns and for lines alone (averaged over four subjects and two target positions) are given in the last column of Figure 1. A single-factor ANOVA showed a significant main effect of pattern on accuracy $[F(3,9)=9.4, p<.005]$. Pattern $A$ produced the highest accuracy: $17 \%$ higher than Pattern $C$, significant by the Newman-Keuls test $(q=33, p<.01)$. The $11 \%$ accuracy difference between Patterns $A$ and $B$ was of borderline significance $(q=20, p=.05)$. Patterns $B$ and $C$ both yielded significantly lower accuracies than lines alone.

\section{Discussion}

The patterns used in this experiment differed significantly in rated depth. The pattern rated highest in 
depth yielded the highest accuracy scores on the line identification task; the pattern rated lowest in depth yielded the lowest scores. The small differences in rated SR are in the opposite direction. These results suggest that the significant differences in accuracy derive from differences in the perceived threedimensionality of the patterns.

It is of interest to note that a pattern high in perceived depth can apparently confer an advantage in identification accuracy even for a target line that is isolated within the pattern-a line that would seem to have "nothing to do" with the rest of the pattern.

\section{EXPERIMENT 2}

A new set of patterns, shown in columns 1 and 2 of Figure 2, was constructed for this experiment. An effort was made to produce as wide a range of perceived depth as possible while holding perceived SR approximately constant. Again, the target line was placed inside the patterns, isolated from the other lines that formed the context.

\section{Method}

Ten new subjects rated the patterns for depth and SR of the target, using the same procedure and the same standard as in Experiment 1. The printed stimuli ranged from 2.8 to $3.1 \mathrm{~cm}$ in width and from 2.4 to $2.6 \mathrm{~cm}$ in height.

Five subjects, three of whom had served in previous experiments, performed the line identification task. The apparatus and procedure were the same as in Experiment 1. The stimuli were from 2.5 to $2.8 \mathrm{~cm}$ in width (1.4 to $1.6 \mathrm{deg}$ of visual angle) and from 2.2 to $2.4 \mathrm{~cm}$ in height (1.3 to $1.4 \mathrm{deg})$; the target line was $.58 \mathrm{~cm}$ long (.3 deg). Luminance levels for the individual subjects ranged from 2.9 to $3.6 \mathrm{~cd} / \mathrm{m}^{2}$.

\section{Results}

Ratings. Mean depth and SR ratings are given in columns 3 and 4 of Figure 2. Single-factor ANOVAs

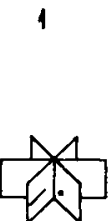

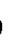
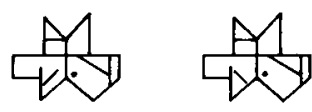

3.3
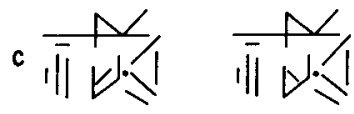

1.4

3.0

53

d

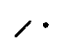$$
\checkmark
$$

(repeated-measures design) were performed on these ratings; the main effect was significant for the depth ratings $[F(2,18)=85.9, p<.001]$ but not for the $S R$ ratings $[F(2,18)=.1]$. The three patterns all differed significantly from each other in rated depth, as shown by Newman-Keuls tests.

Two-factor analyses of covariance (see Footnote 1) revealed no effect of pattern on SR ratings when rated depth was statistically held constant $[F(5,47)=1.0, p>.10]$. The effect of pattern on rated depth remained significant when $S R$ ratings were statistically controlled $[F(5,47)=73.6$, $\mathrm{p}<.001]$.

Accuracy. Mean accuracy scores for the three patterns and for lines alone are given in column 5 of Figure 2. Again, a single-factor ANOVA showed a significant main effect of pattern on accuracy $[F(3,12)$ $=6.5, p<.01]$. Newman-Keuls tests showed that the $16 \%$ accuracy difference between Patterns $A$ and $C$ was significant; $\mathrm{B}$ and $\mathrm{C}$ differed significantly from lines alone.

\section{Discussion}

These three patterns, with their greater total line lengths and their additional diagonal lines, proved quite difficult for our subjects: accuracy for all three patterns was low, compared with the lines-alone condition. Nevertheless, accuracy for the pattern rated highest in depth was significantly above that for the flattest pattern. There was no systematic relationship between the accuracy results and the small differences in rated SR of the target line. Thus, we have shown that differences in perceived depth are sufficient to produce a sizable object-superiority effect, even for an isolated target line. Differences in the perceived SR of the target line are not necessary.

\section{EXPERIMENT 3}

In the previous experiments, the SR of the target line was held relatively constant; in Experiment 3, we attempted to vary it. The aim was to select two threedimensional patterns that differ in the SR of the target line and two flat patterns that also differ in SR.

\section{Method}

Ratings. A set of 18 context patterns was generated. All of these patterns met the criteria of being approximately equal in total line length and in local environment of the target line. A group of 10 new subjects rated these patterns for depth and for SR of the target; the ratings were made in the same manner as in the previous experiments.

The line identification task. The information obtained from the ratings was used in selecting the stimuli for the line identification task. The four context patterns chosen are shown in the first two columns of Figure 3.

Five new subjects (all of them naive as to the purpose of the experiment) performed the line identification task. The apparatus and procedure were the same as in the previous experiments, except that feedback was given visually, on the display screen, after every 10 trials. The stimuli ranged from .8 to $1.3 \mathrm{~cm}$ in width $(.46$ to $.74 \mathrm{deg}$ of visual angle) and from .9 to $1.2 \mathrm{~cm}$ in height (.52 to
Figure 2. The patterns used in Experiment 2 are shown in columns 1 and 2 . Columns 3 and 4 give mean ratings of depth and of structural relevance of the target line. Column 5 gives mean accuracy on the line identification task. 


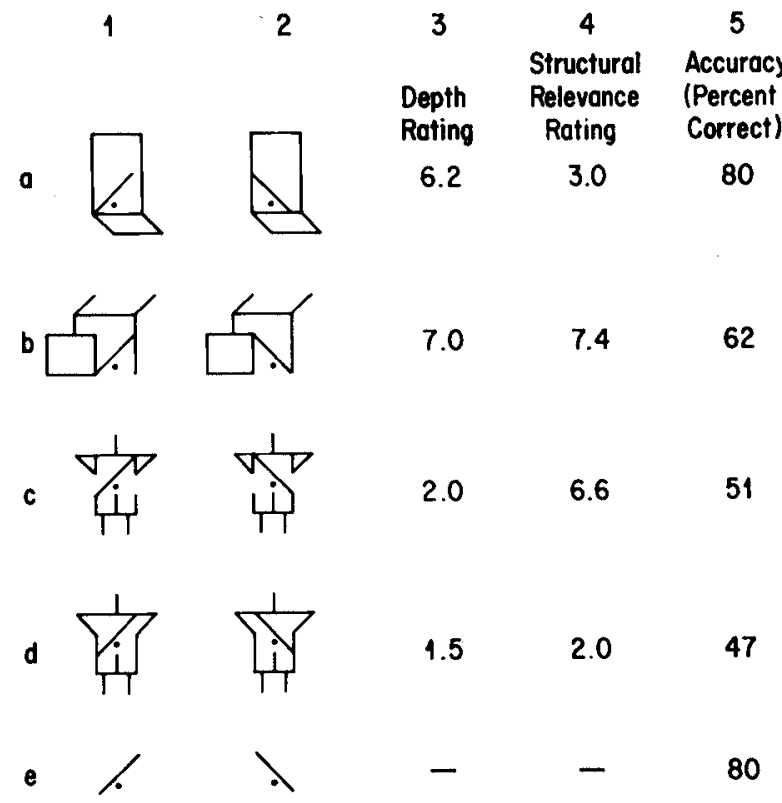

Figure 3. The patterns used in Experiment 3 are shown in columns 1 and 2 . Columns 3 and 4 give mean ratings of depth and of structural relevance of the target line. Column 5 gives mean accuracy on the line identification task.

$.69 \mathrm{deg})$. The diagonal target line was $.6 \mathrm{~cm}$ in length $(.31 \mathrm{deg})$. Display luminance ranged from 3.2 to $3.9 \mathrm{~cd} / \mathrm{m}^{2}$. Each subject served in a total of 240 experimental trials -48 for each context pattern and 48 for lines alone-in random order.

\section{Results}

Ratings. A single-factor ANOVA was performed on the ratings for all 18 patterns. Significant main effects were found both for depth $[F(17,153)=32.3$, $\mathrm{p}<.001]$ and for SR of the target line $[\mathrm{F}(17,153)$ $=14.7, \mathrm{p}<.001]$. Mean ratings for the four context patterns used in the line identification task are given in Figure 3, columns 3 and 4. Newman-Keuls tests on the depth ratings showed that Patterns $A$ and $B$ each differed significantly from $C$ and $D$, but the patterns in those two pairs did not differ significantly from each other. Similarly, Newman-Keuls tests on the SR ratings showed that $A$ and $D$ each differed significantly from B and C; within each pair, the patterns did not differ significantly.

Two-factor analyses of covariance (see Footnote 1) showed a significant effect of pattern on depth ratings when rated SR was statistically held constant $[F(7,63)=23.5, p<.001]$ and a significant effect on SR ratings when rated depth was held constant $[F(7,63)=10.5, p<.001]$.

Accuracy. Mean accuracy scores are given in the last column of Figure 3. A single-factor ANOVA showed a significant effect of pattern on accuracy $[F(4,16)=11.2, p<.001]$. The two patterns rated most three-dimensional yielded the highest accuracies; however, only A differed significantly from the others by a Newman-Keuls test. Accuracy for the three other patterns differed significantly from that in the lines-alone condition.

The two pairs of patterns that differed in rated depth yielded the following mean accuracy levels: $71 \%$ for the high-depth pair and $49 \%$ for the lowdepth pair. On the other hand, the pairs that differed in rated SR yielded these mean accuracies: $57 \%$ for the high-SR pair and $63 \%$ for the low-SR pair. (The latter difference, although small, is in the wrong direction.) A $2 \times 2$ ANOVA on depth (high or low) and SR (high or low) showed a significant effect of depth on accuracy $[F(1,4)=231.6, p<.001]$. Neither the effects of $S R[F(1,4)=6.1, p>.05]$ nor the interaction $[F(1,4)=2.4, p>.05]$ were significant.

\section{Discussion and Overall Results}

Combined results. Experiment 3 showed that a significant difference in perceived SR of the target line, as measured by subjects' judgments of the patterns, is not sufficient to produce an accuracy difference in the line identification task. On the other hand, in all three experiments, differences in rated depth were associated with sizable differences in accuracy. Figure 4 shows the combined results of the three experiments. In these graphs, the mean accuracy score for each context pattern (expressed here as the difference from lines-alone accuracy) is plotted against the mean depth rating (top) and the mean SR rating (bottom) of that pattern. Despite differences in the overall difficulty of the patterns in the three experiments, the correlation between accuracy and rated depth is high $(r=.78, p<.01)$. The correlation with the rated SR of the target line does not differ significantly from zero: $r=-.28$.

Our efforts to vary depth and SR independently in these three experiments were successful: The overall correlation between rated depth and rated $S R$ is $r=$ .01 . Analyses of covariance supported the conclusion that subjects could make independent judgments of the two perceptual variables-SR ratings are not simply unreliable covariates of depth ratings. But SR ratings were less consistent and reliable than depth ratings, even when, as in Experiment 3, the range of ratings was approximately equal (depth range: 5.5; SR range: 5.4). This finding suggests the possibility that the effects of perceived depth may have obscured or covered up the effects of perceived SR. Partial correlation coefficients and a multiple regression analysis provide no support for this interpretation, however. When the effects of rated depth were partialed out, SR ratings were still not significantly correlated with accuracy $(r=-.46, p>.10)$. Partialing out rated SR slightly increased the correlation between accuracy and rated depth $(r=.82, p<.01)$. The multiple regression analysis tested the predictive value of rated depth and rated SR for the 10 patterns used in these experiments. We found a significant overall effect of these variables on accuracy $[F(2,7)$ 


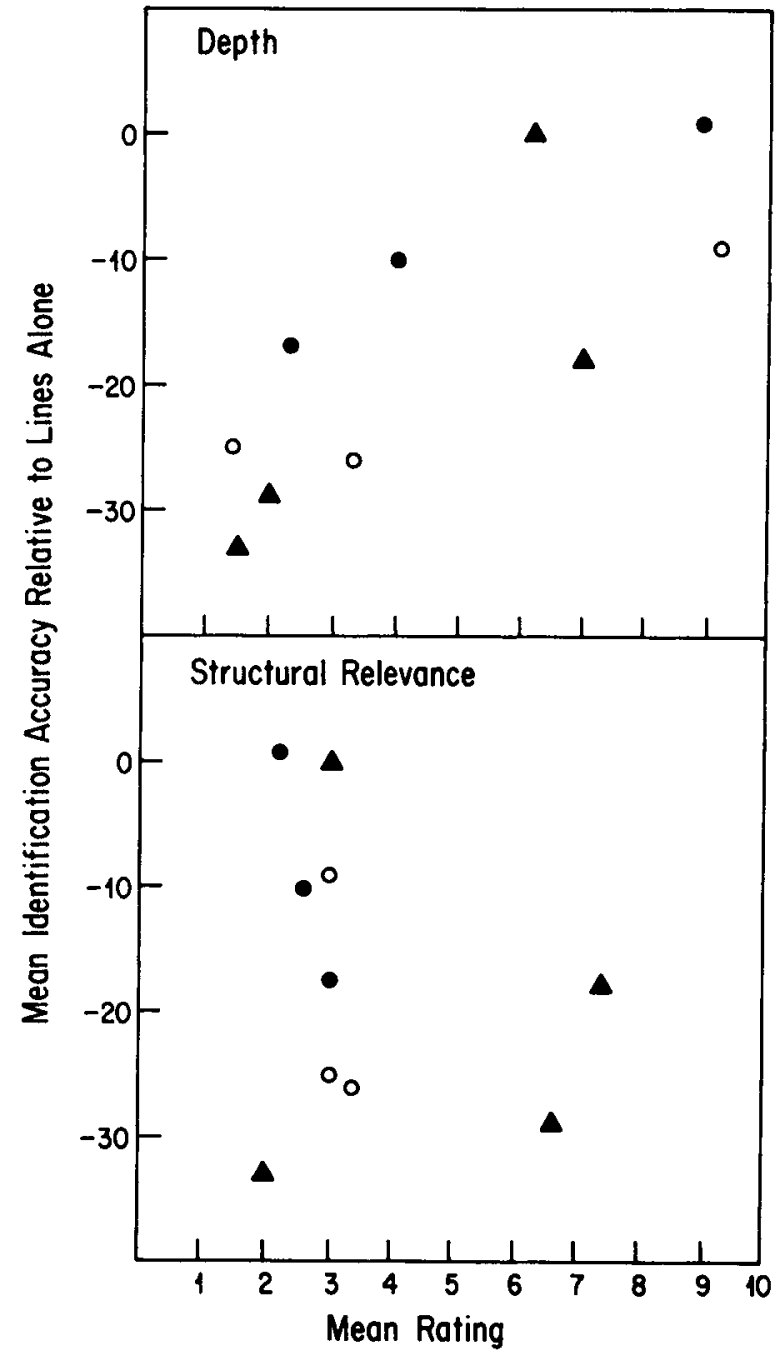

Figure 4. Mean accuracy on the line identification task as a function of rated depth (top) and rated structural relevance of the target (bottom). Accuracy is expressed as difference (In percent correct) from the lines-alone condition. Each point represents one pattern: filled circles, Experiment 1; open circles, Experiment 2; triangles, Experiment 3.

$=7.0, p<.025]$. The regression equation showed that the depth ratings were significant predictors of accuracy $(\mathrm{F}=13.4, \mathrm{p}<.01)$ and accounted for $64 \%$ of the total variance, while the SR ratings were not significant predictors of accuracy $(F=.6)$ and accounted for only $3 \%$ of the variance.

In summary, ratings of perceived depth and perceived SR can be made independently. Identification accuracy was strongly correlated with rated depth, even when the effects of rated SR were statistically controlled. Accuracy was not correlated with rated $\mathrm{SR}$, even when the effects of rated depth were statistically controlled.

Object-line effects. Although strong and significant object-superiority effects were found in these ex- periments, none of the patterns used here produced an object-line effect-accuracy superior to that found with lines alone. These results are consistent with other findings: for instance, Weisstein and Harris (1974) obtained object-superiority effects but no object-line effect. As mentioned above, accuracy declines (all else being equal) as the number and length of lines in the display go up. (Note, for example, the poor overall performance in Experiment 2, which used patterns unusually high in total line length.) Since any facilitative influence of pattern is pitted against the negative influence of the extra lines, the object-line effect is fragile and easily outweighed by any additional negative factors such as placement of the fixation point relative to the lines of the display (Earhard, 1980; Earhard \& Armitage, 1980). This factor may, in fact, have been responsible for our failure to obtain object-line effects in these experiments, since in most of our patterns the fixation point was located near a line or an intersection. However, fixation point location cannot account for the accuracy differences we found among the various patterns we tested. In Experiment 1, for example, fixation point location would predict better performance with Pattern $\mathrm{C}$ than with $\mathrm{A}$ or $\mathrm{B}$. We found the opposite.

Other factors contributing to accuracy differences. The significant accuracy difference found between Patterns $A$ and $B$ in Experiment 3 cannot be accounted for by rated depth, rated SR, or fixation point location. There are other factors, though, that may have contributed to this difference. One is perceived connectedness: Pattern B has some "loose ends"; Pattern A has none. However, other data suggest that connectedness plays, at best, a minor role under the conditions tested here (Weisstein \& C. S. Harris, 1980; Weisstein et al., in press). Another possible factor is what Pomerantz, Sager, and Stoever (1977) have called "emergent features." As those experimenters showed, perceptual features such as triangles and arrows may have a facilitatory effect. In Experiment 3, Variation 1 of Pattern A contains an arrow, and Variation 2 contains a triangle. Pattern $B$ contains neither.

\section{GENERAL DISCUSSION}

In object-superiority effects, a line appearing in certain kinds of patterns is identified more accurately than when it is in other kinds of patterns. It has been claimed that an important aspect of these patterns is how three-dimensional (and, hence, how much like an actual object) they appear to an observer (Weisstein \& Harris, 1980; Weisstein et al., in press; M. C. Williams \& Weisstein, 1981; Womersley, 1977). The alternative claim is that accuracy depends, instead, on the relevance of the target line to the overall 
structure of the pattern (McClelland \& Miller, 1979). Previous attempts to decide between these alternatives have been inconclusive, in part because perceived depth and structural relevance have been confounded.

In the present experiments, perceived depth and perceived structural relevance of the target line were varied independently. The results were clear: identification accuracy was correlated with ratings of perceived depth and not with structural relevance ratings. Even when a line segment appeared as an isolated fragment within a pattern, it was identified more accurately in the patterns perceived as threedimensional. Thus, it seems that the facilitatory influence of a three-dimensional pattern extends across the region bounded by its contours.

We conclude that the object-superiority effect depends on characteristics of the pattern as a whole, rather than on the specific role played within the pattern by the target line. The probable source of this effect, and of related effects (Weisstein \& Maguire, 1978; M. C. Williams \& Weisstein, 1981) is the visual system's ability to respond more rapidly ${ }^{2}$ to certain kinds of stimuli-stimuli that appear threedimensional, well structured, unified, and somewhat redundant-in short, stimuli that look like objects.

\section{REFERENCE NOTES}

1. Wandmacher, J. Personal communication, 1979.

2. Ozog, G. Unpublished experiment (cited in Weisstein \& Harris, 1974).

3. Genter, C. R., II, \& Weisstein, N. A temporal objectsuperiority effect. Manuscript submitted for publication, 1981.

4. Wong, E., \& Weisstein, N. Signal integration functions for targets in object-superiority patterns. Manuscript submitted for publication, 1981.

\section{REFERENCES}

Earhard, B. The line-in-object superiority effect in perception: It depends on where you fix your eyes and what is located at the point of fixation. Perception \& Psychophysics, 1980, 28, 9-18.

Earhard, B., \& Armitage, R. From an object-superiority effect to an object-inferiority effect with movement of the fixation point. Perception \& Psychophysics, 1980, 28, 369-376.

Genter, C. R., II, \& Weisstein, N. Perceived depth and connectedness affect flicker frequency function. Investigative Ophthalmology and Visual Science, 1980, 19(Suppl.), 105. (Abstract)

KLEIN, R. Visual detection of line segments: Two exceptions to the object superiority effect. Perception \& Psychophysics, 1978, 24, 237-242.

McClelland, J. L., \& Millen, J. Structural factors in figure perception. Perception \& Psychophysics, 1979, 26, 221-229.

Pomerantz, J. R., Sager, L. C., \& Stoever, R. G. Perception of wholes and their component parts: Some configural superiority effects. Journal of Experimental Psychology: Human Perception and Performance, 1977, 3, 422-435.

WEISSTE IN, N., \& Harris, C. S. Visual detection of line segments: An object-superiority effect. Science, 1974, 186, 752-755.

Weisstein, N., \& Harris, C. S. Masking and the unmasking of distributed representations in the visual system. In C. S. Harris (Ed.), Visual coding and adaptability. Hillsdale, N.J: Erlbaum, 1980 .

Weisstein, N., \& Maguire, W. Computing the next step: Psychophysical measures of representation and interpretation. In A. Hanson \& E. Riseman (Eds.), Computer vision systems. New York: Academic Press, 1978.

Weisstein, N., Williams, M. C., \& Harris, C. S. The role of perceived depth in object-superiority effects: Line segments are harder to see in flatter patterns. Perception, in press.

Weisstein, N., Williams, M. C., \& Williams, A. Connectedness and three-dimensionality affect different aspects of the metacontrast function. Investigative Ophthalmology and Visual Science, 1979, 18(Suppl.), 1. (Abstract)

Williams, A., \& Weisstein, N. The time course of object superiority with contexts whose local environments are similar. Bulletin of the Psychonomic Society, 1977, 10, 243. (Abstract)

Williams, A., \& Weisstein, N. Line segments are perceived better in a coherent context than alone: An object-line effect. Memory \& Cognition, 1978, 6, 85-90.

Williams, M. C., \& Weisstein, N. Spatial frequency response and perceived depth in the time-course of object superiority. Vision Research, 1981, 21, 631-646.

Womersley, M. A contextual effect in feature detection with application of signal detection methodology. Perception \& Psychophysics, 1977, 21, 88-92. were pattern (six pattern variations in Experiments 1 and 2, and eight in Experiment 3) and order in which the ratings were made (depth first or SR first). The analyses examined the effects of these two variables on depth ratings and SR ratings. Neither order of ratings nor the interaction between order and pattern yielded significant effects in any experiment.

2. Results from metacontrast (Weisstein \& Harris, 1980; M. C. Williams \& Weisstein, 1981) and flicker experiments (Genter \& Weisstein, Note 3) and from free reaction-time procedures and signal integration functions (Wong \& Weisstein, Note 4) indicate that, overall, visual response is faster to target lines in patterns judged three-dimensional than to lines in flatter patterns. However, speed-accuracy data obtained by Wong and Weisstein (Note 4) show that, while rise time is faster for targets in objectlike contexts, latency is somewhat longer. In other words, it seems that visual response to three-dimensional patterns actually begins later than the response to flat patterns, but, once begun, it gains amplitude more rapidly and reaches a higher asymptote. The longer latency may account for Klein's (1978) seemingly contradictory observation of a breakdown of object-superiority effects under "deadline" response conditions: if subjects must respond before some minimal threshold criterion is reached, neither speed nor accuracy is likely to be greater for object-like patterns.

(Manuscript received February 23, 1981; revision accepted for publication January $15,1982$. 